\title{
Mapping Non-Destructive Testing Data on the 3D Geometry of Objects with Complex Shapes
}

\author{
by S. Soldan*, D. Ouellet**, P.Hedayati**, A. Bendada**, D. Laurendeau**, A. Kroll*
}

*University of Kassel, Measurement and Control Department, Germany, http://www.uni-kassel.de/fb15/mrt

**Laval University, Department of Electrical and Computer Engineering, Canada, http://www.gel.ulaval.ca

\begin{abstract}
This paper presents an approach for measuring the geometry of objects with complex shapes with a 3D camera and, at the same time, collecting surface and subsurface information that is then mapped onto the geometry in good spatial alignment. Subsurface information can be captured with infrared thermography or ultrasonic probes. The approach can thus allow non-destructive testing procedures to be used for detecting, locating and measuring the size, shape and depth of undersurface defects of an object and to map these defects, and potentially other information such as reflectance or color, onto the geometric model of the object.
\end{abstract}

\section{Motivation}

In a long-term monitoring context, the inspection of objects with complex geometries using non-destructive techniques such as thermal infrared imaging or phased array ultrasound sensing implies that the undersurface structure of the object to be observed is in good registration with the 3D object geometry. This paper presents an approach for measuring the geometry of objects with complex shapes using a handheld 3D scanner and for collecting subsurface information, in this case thermal images or ultrasonic recording, in registration with the captured object geometry. An important step leading to accurate registration of thermal and geometric data is the calibration of the intrinsic and extrinsic parameters of the pinhole camera model used to model the infrared camera. Intrinsic parameters describe "internal" properties of the camera such as focal length, principal point, radial/tangential distortion, etc. Extrinsic parameters describe the position and orientation (i.e. the pose) of the pinhole in a global reference frame (often called the "world" reference frame). Once calibration is complete, the "storage" of thermal data in the 3D map can be achieved with the help of a motion tracking system.

The paper is organized as follows. The experimental setup and the various instruments used for the experiments are described in Section 2. Second, the model that was adopted for describing image formation by the thermal infrared camera is described briefly in Section 3. Then, the procedure for calibrating the pinhole modelling the infrared camera is described in detail and validation experiments are presented and discussed in Section 4 . Section 5 explains the procedure that is adopted for acquiring and registering $3 \mathrm{D}$ and thermal data. Experimental results are presented. Section 6 presents an extension of the thermal mapping approach to the case of phased array ultrasound data. Finally, conclusion and perspectives of future work are given in Section 7.

\section{Experimental setup and description of the instruments}

Fig. 1 shows the different elements composing the experimental setup that was used for mapping thermal infrared data on the 3D model of an object or scene. The main instruments of the setup consist of $i$ ) a handheld 3D scanner, ii) an infrared thermal camera, and iii) a motion tracking system. The object or scene of interest is placed on a support and tracking markers are installed on its surface. It is to be noted that, as discussed later in Section 4, the scene of interest may be the object to be scanned or the calibration target. Tracking markers are also installed on the infrared camera case. The world reference frame, labeled $O_{V}$, is chosen to be the one defined by the motion tracking system. A reference frame $\left(O_{C}\right.$ in Fig. 1$)$ is attached to the case of the infrared camera. A third reference frame, $O_{P H}$, is attached to the pinhole modelling the image acquisition process of the infrared camera.

The motion tracking system provides accurate estimates of the pose of the object and of the camera case in the world reference frame through frame transformation matrices $T_{V B}$ and $T_{V C}$ respectively. Transform $T_{P H-B}$ between the reference frame of the pinhole, $O_{P H}$, and the reference frame of the calibration target, $O_{B}$, is estimated according to the procedure described in Section 4. Transform $T_{C-P H}$ between the reference frame of the camera case $\left(O_{C}\right)$ and the reference of the pinhole $\left(O_{P H}\right)$ is computed from the other transforms. There is no need to estimate the frame transformation between the handheld scanner and the motion tracking system or the transform between the scanner and the object/scene since this is taken care of automatically by the scanner which positions itself with respect to the markers installed on the object/scene.

The thermal camera used for the experiments is a Jenoptik IR-TCM 384 with the specifications listed in Table 1. The camera is radiometrically calibrated and reports the incoming radiation as a temperature in Kelvin. It can perform automatic non-uniformity correction (NUC) with an inbuilt shutter and is equipped with a motorized focus. A software development kit (SDK) controls the camera. 


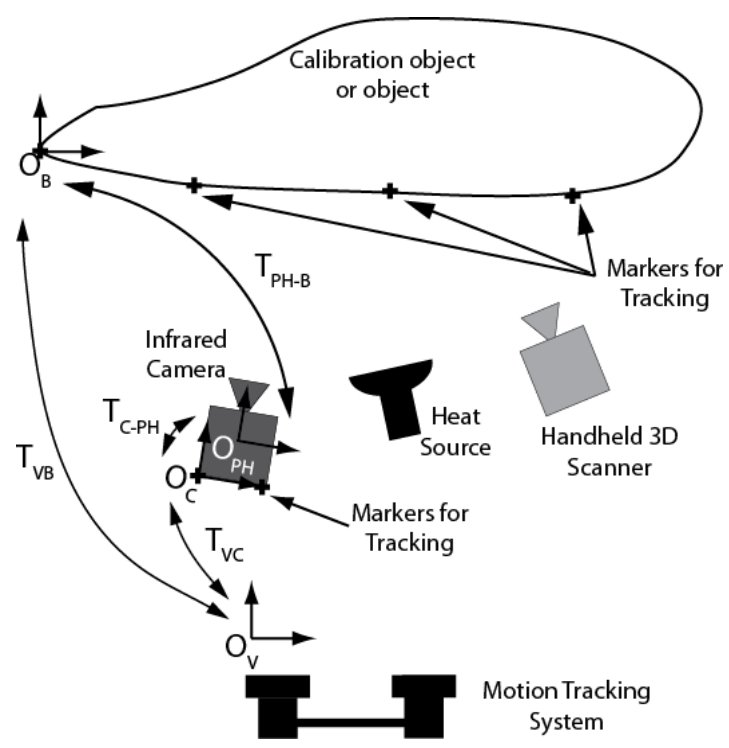

Fig. 1. Experimental setup and reference frames involved in the experiments

The focus motor is controlled with focus steps (or motor increments) $d_{v, \text { CAM }}$ from 0 to 1180 (only even numbers are used). The relation between focus steps $d_{v, \text { CAM }}$ and the distance between the lens and the image sensor $d_{v}$ is described by an affine transformation:

$$
\mathrm{d}_{\mathrm{v}}=\mathrm{a} \mathrm{d}_{\mathrm{v}, \mathrm{CAM}}+\mathrm{b}
$$

The focal distance $d_{u}$ is given by:

$$
d_{u}=\left(f^{-1}-d_{v}^{-1}\right)^{-1}
$$

The relationship between the focus steps and the focal distance as well as the relationship between $d_{v}$ and $d_{u}$ are plotted in Fig. 2.

\begin{tabular}{|l|l|}
\hline Pixel resolution & $384 \times 288$ pixels \\
\hline Spectral range & $7.5-14 \mu \mathrm{m}$ \\
\hline Lens focal length $f$ & $25 \mathrm{~mm}$ \\
\hline Lens f-number & 1 \\
\hline Detector pitch & $35 \mu \mathrm{m}$ \\
\hline Field of view & $30^{\circ} \times 23^{\circ}$ \\
\hline Connection with host PC & FireWire and GigE \\
\hline
\end{tabular}
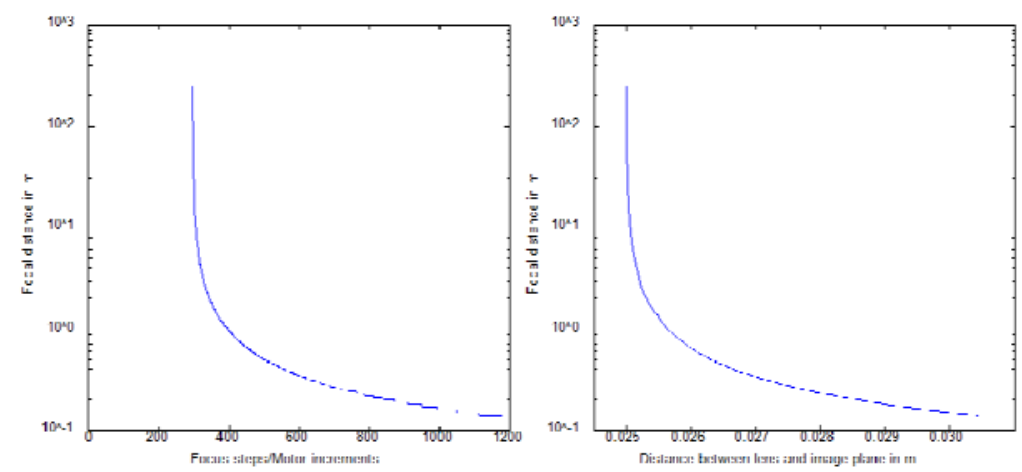

Fig. 2 Focal distance vs camera focus step (left) and focal distance vs distance between lens and image plane (right) 
A commercial 3D scanner, the Creaform Go!SCAN 3D [2], is used for measuring the 3D geometry of the object/scene. The handheld scanner has to be manually moved around the object to collect the 3D data but does not have to follow a specific path other than that the motion should be smooth. The scanner is geometrically calibrated and reports the object dimensions in millimeters. The pose of the scanner with respect to a reference frame in which the 3D measurements are defined is estimated using geometric features of the object (e.g. corners, edges, etc.) as well as flat circular self-adhesive retro-reflective markers installed on the object/scene. The markers improve the stability of the scanning process, especially in regions with little features (e.g. an outstretched pipe). These markers must not be confused with the tracking markers illustrated in Fig. 1. Model resolution can be as small as $0.5 \mathrm{~mm}$. The VXelements control software and GUI guides the user during acquisition and post-processing of the data. VXElements offers postprocessing functionalities like surface reconstruction/optimization, hole filling, cleaning of outliers, etc. The output of the scanner is a triangulated mesh and the positions and normal vectors of the reflective markers. This data can be saved in various ASCII and binary standard formats. By default, the reference frame of the 3D data is defined by the position and orientation of the scanner in the first frame of the scan. However the software provides advanced options to change the coordinate system and align it with points, planes or lines in the 3D model.

Fig. 3 shows the metal pipe structure used for the experiments presented in this paper. Retro-reflective markers are installed on the pipe structure and the table supporting the structure. The shaded 3D model (mesh) obtained with the handheld scanner is shown on the right hand side of the figure.
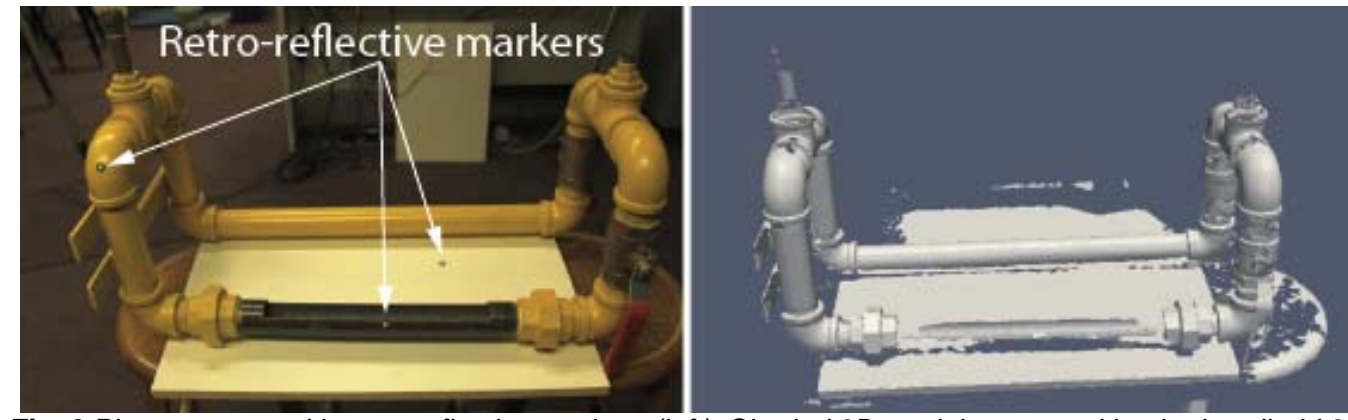

Fig. 3 Pipe structure with retro-reflective markers (left). Shaded 3D model measured by the handheld 3D scanner (right)

The tracking system is composed of a set of eight Vicon T-Series cameras installed so as to observe the workspace around the object/scene. The position of the retro-reflective markers (the tracking markers) shown in Fig. 1 can be precisely tracked (accuracy better than $1 \mathrm{~mm}$ ) in a volume of approx. $3 \times 3 \times 2 \mathrm{~m}^{3}$ at high frame rates $(>100 \mathrm{~Hz})$ and is reported in $\mathrm{mm}$. By grouping more than three rigidly coupled markers on an object, the orientation of the object can be tracked in addition to its position. The motion tracking system uses a graphical user interface ('Vicon Tracker') to configure objects and stream their pose (position + orientation) via a network protocol. This pose information is used by our application to map infrared thermal data on the 3D model.

The Vicon system is calibrated by moving a known object (T-wand) in the workspace covered by the cameras. The tracking software then automatically calculates the camera positions and optical parameters. To set the origin of the tracking coordinate system the wand object is used as well. The positions reported by the motion tracking system are used as a global reference. The Vicon system usually uses spherical reflective markers (different diameters are available) but can also track the flat markers used by the 3D scanner if they are nearly perpendicular to the cameras' view axis. Since the motion tracking system assumes spherical markers, the position of a flat marker is reported to be around $1 \mathrm{~mm}$ below the surface of the marker. It is relevant to mention that, contrarily to the Vicon which can use the 3D scanner markers for pose estimation, the handheld scanner cannot use the tracking markers for self-positioning.

\section{Infrared camera model and calibration target}

Image formation for the infrared thermal camera is modeled by a non-inverting pinhole. As shown in Fig. 4, in this model, a point $M$ in space is projected as point $m$ on the image plane through a straight ray $M-m-C$, where $C$ is the projection center. The pinhole reference frame (frame $O_{P H}$ in Fig. 1) is located at $C$ with the optical axis along axis $Z$ and normal to the image plane. The optical axis intersects the image plane at the principal point $P$.

In order to achieve the mapping of infrared image data on the 3D geometric model described in Section 1 , parameters of the pinhole model must be calibrated. The necessary intrinsic parameters for this model are the focal length in pixels $\left(f_{x}, f_{y}\right)$ (calculated from focal length in $m$ divided by pixel pitch in $m / p x$; for cameras with lenses this value changes with focus and appears to better resemble the distance between the image plane and the lens/pinhole than the fixed focal length), the principal point in pixels $\left(c_{x}, c_{y}\right)$ which is the center of the detector chip, and the skew coefficient which describes the angle between the horizontal and vertical axes of the sensor and which is usually 0 for digital cameras. 
A point $[x, y, z]$, in the camera coordinate system with the projections $x^{\prime}=x / z$ and $y^{\prime}=y / z$ will appear at pixel coordinates $u=f_{x} x^{\prime}+c_{x}$ and $v=f_{y} y^{\prime}+c_{y}$. Note that camera pixels are numbered from $(0,0)$ in the top left corner of the image plane (sensor).

In order to take into account the distortion caused by the camera lens, radial and tangential distortion parameters are also included in the set of intrinsic parameters. The equations modelling distortion are:

$$
\begin{aligned}
& x^{\prime \prime}=x^{\prime}\left(1+k_{1} r^{2}+k_{2} r^{4}+k_{3} r^{6}\right)+2 p_{1} x^{\prime} y^{\prime}+p_{2}\left(r^{2}+2 x^{\prime 2}\right) \\
& y^{\prime \prime}=y^{\prime}\left(1+k_{1} r^{2}+k_{2} r^{4}+k_{3} r^{6}\right)+p_{1}\left(r^{2}+2 y^{\prime 2}\right)+2 p_{2} x^{\prime} y^{\prime}
\end{aligned}
$$

where $r^{2}=x^{\prime 2}+y^{\prime 2}$. Although higher distortion models could be used, this paper considers the five parameters $\left(k_{1}, k_{2}, k_{3}\right.$, $\mathrm{p}_{1}, \mathrm{p}_{2}$ ) in Eq. (3).

All model parameters are determined through a calibration procedure to be described in Section 4. A calibration target (usually a planar object) with features of known shape and dimensions is used to acquire multiple observations of the features (see Fig. 5.). The number of observations roughly depends on the number of features comprised on the calibration target and the size of the workspace of the camera to be covered.
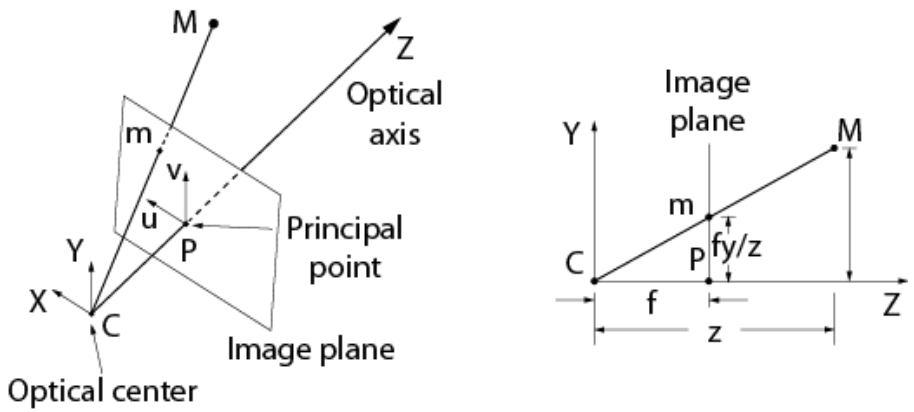

Fig. 4 Pinhole model for the infrared camera showing the center of projection $C$, principal point $P$, image plane, and projection ray (left). Side view of the projection geometry (right)

Several types of targets for the calibration of infrared thermal cameras have been proposed in the literature. A survey of the different targets is available in [3]. Some targets are active, meaning that the features are heated in order to make them easier to detect in the image, while others are passive.

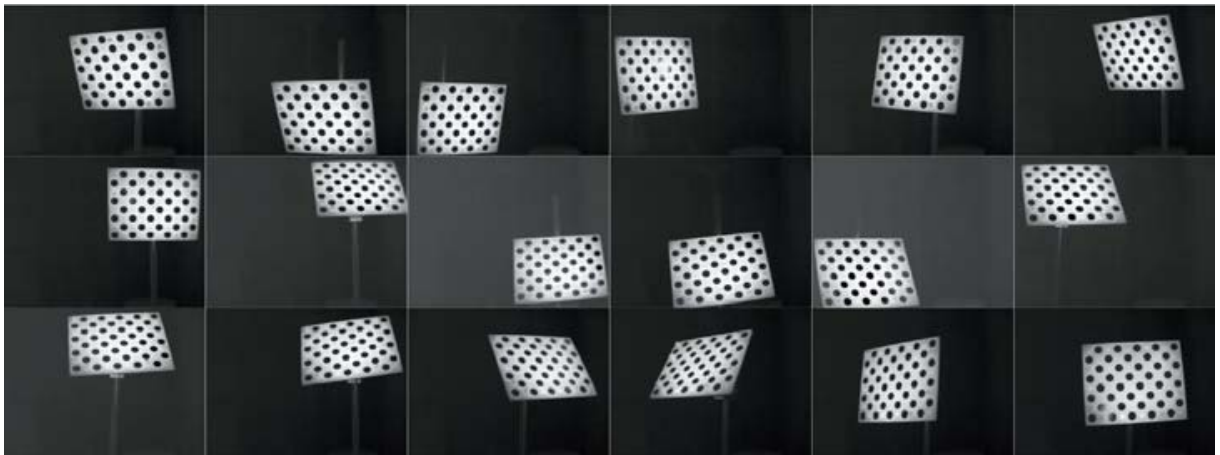

Fig. 5 Subset of 18 images from a calibration series of 21 observations using a thermal camera and a calibration object made of circles arranged as a pattern of features

The target that was used for the experiments reported in this paper is shown in Fig. 6. Based on the literature review it was decided to use a passive target since active targets seem to require more manual work to manufacture, potentially resulting in a lower accuracy. It was decided to fabricate a printed circuit board (PCB)-based calibration target. Since PCB machining is executed under computer control, fabrication accuracy is expected to be very high. A two-sided copper PCB with two different structured patterns was chosen: a matrix of evenly spaced circles on one side and a chessboard on the other. The advantage of these patterns is that robust feature detectors (circles or corners of squares) with sub-pixel accuracy are available [4]. Heating the target with a source of energy (hairdryer for instance) allows the features to be made clearly visible in the infrared image (see Fig. 5). In short, the target as such is passive but can be made "active" by using an external heat source. The target thus combines the simplicity of fabrication of passive targets with the advantages of active ones. 


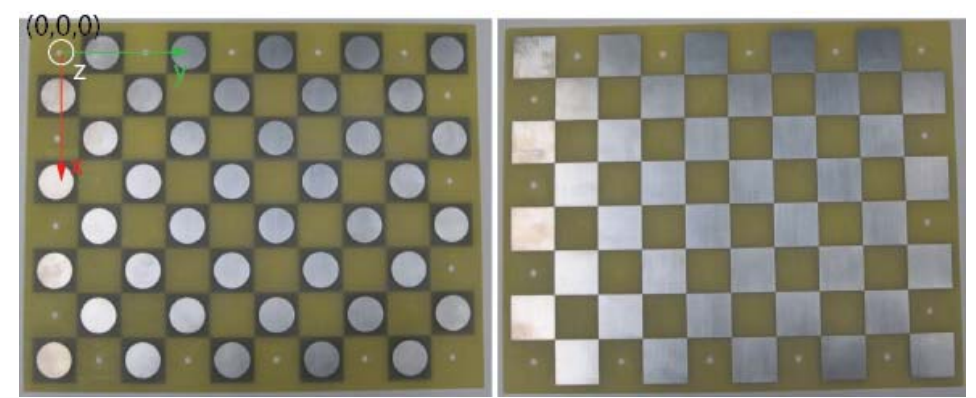

Fig. 6 Calibration target used for the experiments. The pattern is made of a matrix of circles on one side of the target, while the other side is made of a chessboard of squares. The PCB material is opaque to the 3-5 $\mu \mathrm{m}$ and 7-14 $\mu \mathrm{m}$ spectral bands.

Experiments have shown that better results were achieved with the circles pattern which was less sensitive to lens defocusing and which led to more accurate estimation of the intrinsic and extrinsic parameters. For this reason, the circles pattern was chosen for the calibration experiments reported in Section 4.

As shown in Fig. 1, tracking markers are installed on the calibration target (not visible in Fig. 6). The objective for placing these markers on the calibration target is to be able to determine the position of the features (corners, circle centers) in the 3D motion tracker coordinate system. This also allows to estimate transform $T_{C-P H}$ between the reference frame attached to the camera case and the reference frame of the pinhole model (see next section).

\section{Infrared camera calibration procedure and validation experiments}

The calibration process consists of the determination of the intrinsic and extrinsic camera parameters. Calibration proceeds as follows. Images of the calibration target are acquired at different positions and orientations with respect to the camera (see Fig. 5). The features of the target are detected in each image and are matched with the ones on the actual target for which the position are known in the reference frame of the target. Intrinsic and extrinsic parameters are finally calculated with a closed-form solution and improved using non-linear optimization. The information that has to be recorded for each observation is therefore: $i)$ the position of features of the calibration target in pixel coordinates, ii) the position of the features in object coordinates, iii) the global position of the target features and iv) the global position of an arbitrary placed coordinate system attached to the camera. Since the intrinsic camera calibration depends on the focus settings of the camera, these settings must remain constant for the duration of the calibration process and, once calibrated, when the camera is used for experiments.

The detection of features on images of the calibration target is comprised of several steps. First, infrared thermal image data is obtained from the camera and converted to 8-bit greyscale image data with automated color scaling (minimum temperature $\rightarrow$ black, maximum temperature $\rightarrow$ white; linear scaling). Then, using OpenCV [4] functions, the features of the calibration target are detected if the target is present in the field of view (FOV) of the camera and the features are clearly detectable. Third, the image is displayed on a computer screen and the features are augmented if they have been detected (Fig. 7). If the visibility and position of the target is satisfactory, the list of the feature coordinates in pixels are recorded. This procedure has to be repeated around 20 times to cover the whole FOV of the camera and also to exhibit different viewing angles with respect to the optical axis. Once the aforementioned information has been collected, the OpenCV optimization algorithm ${ }^{1}$ is run and returns the reprojection error (which consists of the RMS error between the image coordinates of the detected features and the coordinates of the projection of the target features using the estimated camera parameters), camera matrix, distortion parameters and the pose $T_{P H-B}$ of the calibration target (for each observation) with respect to the camera reference frame (see Fig. 1). The interested reader is referred to [5] for details on the calibration procedure.

Once the above steps are completed, the next step is to determine the pose of the pinhole model reference frame $O_{P H}$ in the global reference frame $\left(O_{V}\right)$. In order to find this transform, transform $T_{C-P H}$ must first be obtained. Using the tracking system, the pose of the calibration target is known in the reference frame of the tracking system ( $\left.T_{V B}\right)$ and of the pinhole reference frame $\left(T_{P H-B}\right)$. One can then write for $T_{C-P H}$ :

$$
\mathrm{T}_{\mathrm{C}-\mathrm{PH}}=\mathrm{T}_{\mathrm{VC}}{ }^{-1} \mathrm{~T}_{\mathrm{VB}} \mathrm{T}_{\mathrm{PH}-\mathrm{B}}{ }^{-1}
$$

Consequently, a point with coordinate $x_{P H}$ in the reference frame of the pinhole model has coordinate $x_{V}$ in the global reference frame given by:

$$
\mathrm{X}_{\mathrm{V}}=\mathrm{T}_{\mathrm{VC}} \mathrm{T}_{\mathrm{C}-\mathrm{PH}} \mathrm{X}_{\mathrm{PH}}
$$

so:

\footnotetext{
${ }^{1}$ Calibration function cv2.calibrateCamera
} 


$$
\mathrm{X}_{\mathrm{PH}}=\mathrm{T}_{\mathrm{C}-\mathrm{PH}}{ }^{-1} \mathrm{~T}_{\mathrm{VC}}{ }^{-1} \mathrm{XV}_{\mathrm{V}}
$$

with $T_{C-P H}$ obtained from Eq. (4). Eq. (5) allows the computation of the coordinates of a point in the reference frame of the pinhole if its coordinates are known in the global reference frame.

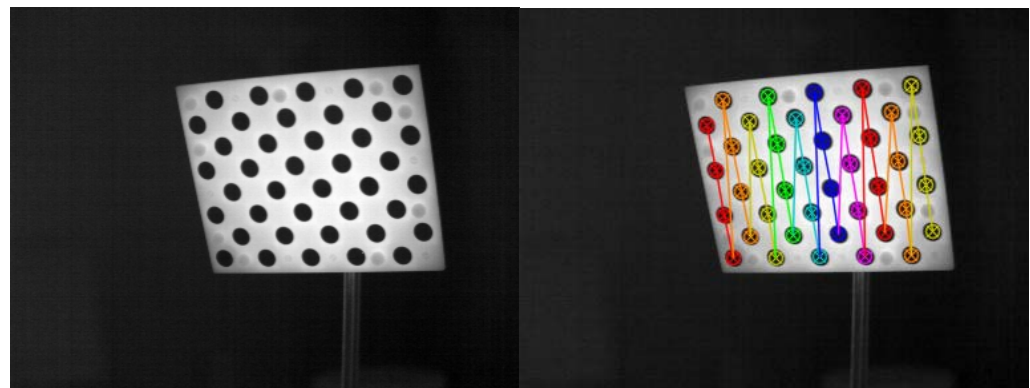

Fig. 7 Calibration target seen by the thermal camera (left, min. temp. $294.76^{\circ} \mathrm{K}$, max. temp. $303.83^{\circ} \mathrm{K}$ ) and detected features (right)

Validation experiments have shown that, using the parameters estimated by the calibration procedure and the transforms provided by the tracking system, the reprojection error of the discs on the calibration target was around 0.35 pixels on average.

Experiments have also been performed to assess the influence of focal settings on calibration parameters. As mentioned above in Section 2, it is possible to change the focal length of the infrared thermal camera using the focal motor. In these experiments, the calibration was performed as described previously but instead of taking only one measurement for each observation, the focus was changed to certain values $d_{v, \text { CAM }}$ and one measurement was taken for each (the position of the camera and the calibration object remained unchanged while the focus was changed and the images where captured). Following acquisition, calibration parameters were estimated for each focus value. The conclusion of these experiments was that the principal point and the position and orientation of the pinhole reference frame (except for the $z$ position) seem to be constant for different focus settings. All other parameters (focal length, distortion parameters, position of the pinhole reference frame in the $z$-direction) change for different focus settings. It was also found that the parameter changes are not looking very predictable so it seems difficult to define a mathematical model that would allow the parameters to be obtained analytically. It would rather be best to empirically create a lookup table to select the appropriate parameter set.

\section{3D and thermal data acquisition/registration procedure and experimental results}

With the setup shown in Fig. 1 calibrated, it is possible to capture infrared thermal images and 3D images and then map the thermal information on the 3D geometry. The semi-automatic workflow shown in Fig. 8 has been implemented to achieve this goal.

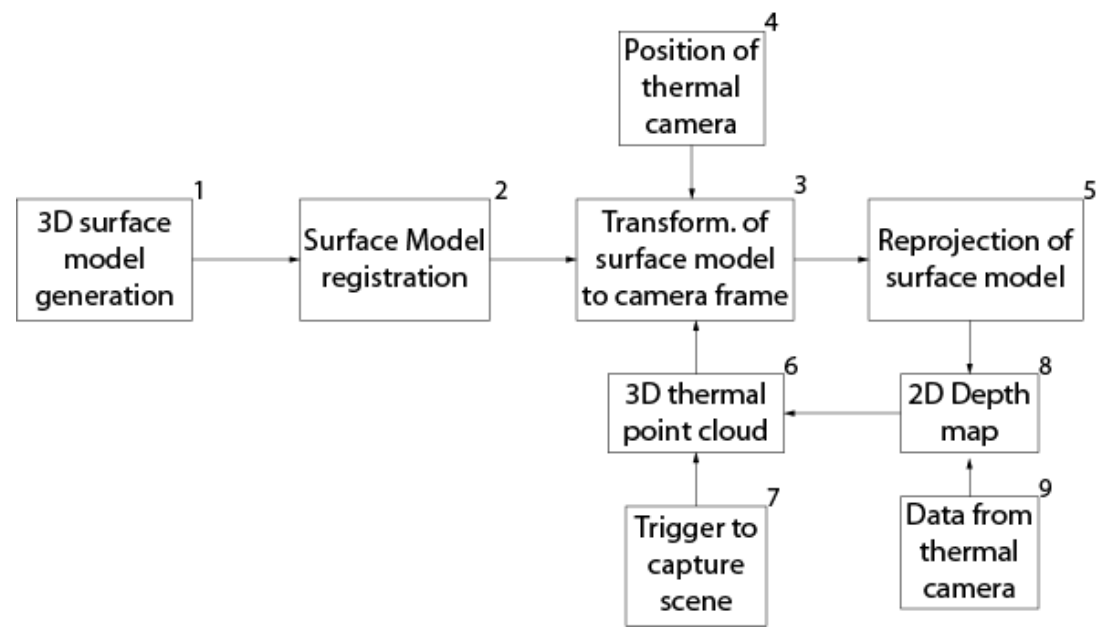

Fig. 8 Workflow of the $3 D$ acquisition and registration process

3D model generation (box 1 on Fig. 8) is concerned with the measurement of the geometry of the object/scene of interest. The 3D surface model is recorded by slowly moving the handheld 3D sensor around the object and thereby 
scanning/sweeping the object's surface. As mentioned earlier, retro-reflective markers are used to support sensor selflocalization and improve the quality of the collected 3D data. The recorded surface data is enhanced by removing artifacts and filling small holes (this is done manually with the software provided by Creaform) and the geometry is exported to $a *$.ply file. The position and surface normal of the markers are also exported.

The registration of the 3D model and the global reference frame (box 2 on Fig. 8) can either be done via mutual retro-reflective markers or by aligning the coordinate systems manually in the software of the 3D scanner (if the global coordinate origin and orientation is attached to geometric objects, e.g. the corner of a table). Both approaches were tested and work equally well. Using the retro-reflective markers has a slight advantage since it allows the object to be moved without re-referencing the motion tracking system.

The transformation of the surface model in the camera reference frame (box 3 and 4 on Fig. 8) is performed by Eq. (6) using the data obtained at the calibration step.

The next step consists of reprojecting the surface model to the thermal camera perspective (box 5 in Fig. 8). At this step, the goal is to determine which part of the surface model is visible from the thermal camera point of view so the thermal information can be mapped onto the corresponding 3D points. To achieve this task, the surface model is transformed into the pinhole model reference frame and the data can be either reprojected or the lines of sight (or beams/rays) for each thermal camera pixel can be traced up to the surface model to figure out which element of the 3D model is hit by the ray. It was decided to reproject the 3D model since that seemed to be the faster approach (in terms of processing time). The resulting reprojection is shown in Fig. 9. In this figure, 3D surface points are colored based on the distance between the camera and the surface model and basically shows what the camera would report if it were a depth camera instead of a thermal camera. With this depth information, 3D points in the pinhole model reference frame can be identified for each pixel.

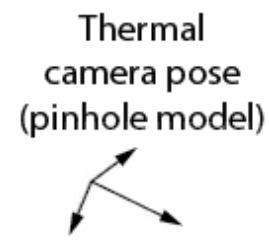

Fig. 9 Surface model with camera position indicated in the top-left corner. The points shown are those points on the surface that are seen from the camera point of view. The color indicates the distance from the camera (blue: close, red: far)

Once the mapping between thermal pixels and 3D points has been performed for each thermal camera view, all that remains is to transform the 3D points back in the global reference coordinate frame and to save 3D points with the corresponding temperature as an attribute. Other attributes could also be stored such as frame number, angle between surface and camera optical axis, distance to camera. For data storage, the XML based VTK *.vtu [6] format was used to be opened with compatible viewers (e.g. ParaView [7] or KiwiViewer [8]) or converted to other formats if needed.

To test the mapping, the pipe structure shown in Fig. 3 was heated at one end (in order to generate a visible heat contrast with respect to other parts of the structure) and thermal infrared images were captured according to the process described above. Image data was recorded from six different camera poses. The resulting geometric model with thermal data mapped on the surface can be seen in Fig. 10 and illustrates that the described process works well. 


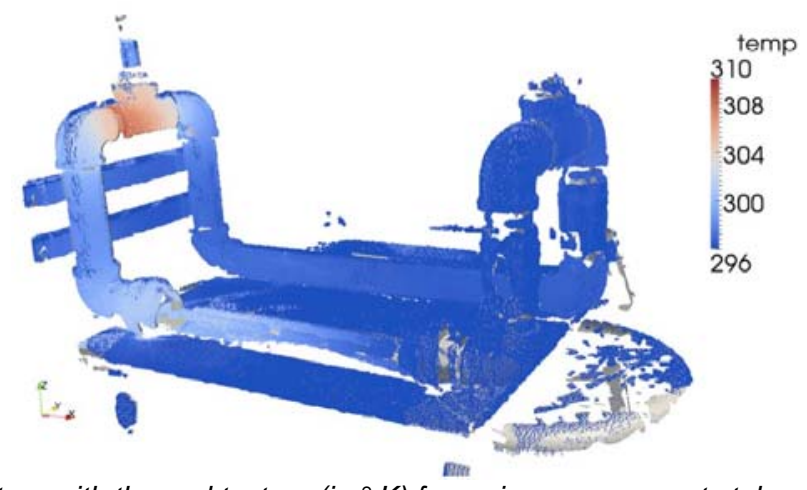

Fig. $103 D$ pipe structure with thermal texture (in ${ }^{\circ} \mathrm{K}$ ) from six measurements taken from different camera positions. The left hand part was heated with an external heat source.

In Fig. 11, the same 3D model is colored based on the number of the point of view from which it was taken by the thermal camera and shows that the data is mosaicked from different camera poses.

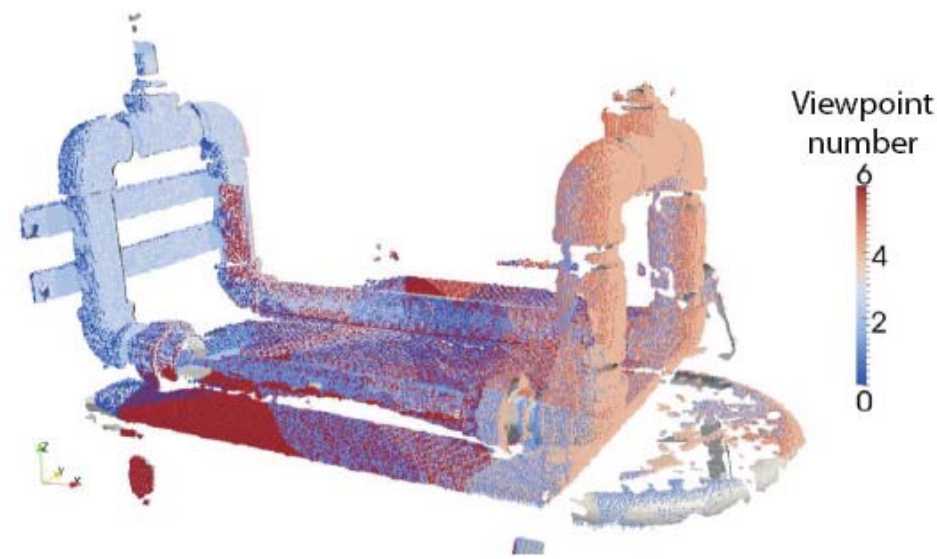

Fig. 11 3D Pipe model with colors indicating from which point of view the thermal measurement was captured

These results show that the proposed method is able to achieve a good alignment between the thermal maps and the 3D model and that the mapping of thermal information on the 3D structure allows the observation of this information on the actual geometric structure on which it is captured.

\section{Extension of the registration approach to phased-array ultrasonic data}

The above mapping method has been extended to the case of non-destructive testing using a phased-array ultrasonic transducer. In this case, tracking markers were installed on the ultrasonic transducer (similarly to the case of the thermal infrared camera). The transducer was linked to an Olympus OmniScan MX which was connected via a network cable to a Windows PC running TomoView 2.10R2 as well as NDT RemotControlLibrary 2.6R18. In this case, the transformation between the ultrasonic transducer and the global reference frame was calibrated and the subsurface measurements obtained by sweeping the transducer on the object to be inspected were mapped on the 3D model of the object. Fig. 12 shows several aluminium/iron flat plates with simulated defects and the result of the mapping of the ultrasonic data on their geometry (left). The ultrasonic scans were collected on the smooth side of the plates (Sonoglide coupling gel was used). The results show a great resemblance of the defects. The measured depths were confirmed with a caliper to be very accurate. Because these objects are plates, they do not show the full potential of the approach. Therefore additional curved objects were used and can be seen in Fig. 12 (right). 

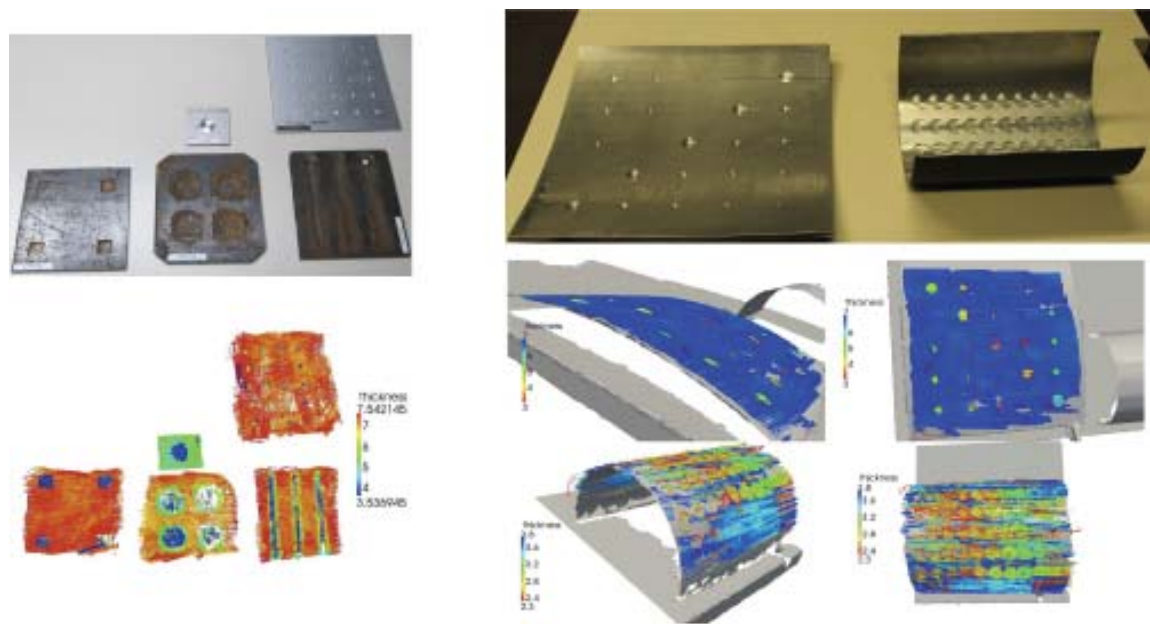

Fig. 12 Mapping of ultrasonic data on the 3D geometry. Flat plates (left), cylindrical plates (right)

\section{Conclusion and future work}

In this paper, an approach for mapping infrared thermal data or ultrasonic data on the 3D model of an object has been presented. As demonstrated in the experiments, the approach provides interesting results. Future work will address several issues. First, accuracy has been considered in many facets (motion tracking accuracy, 3D scanning accuracy, 3D surface model registration accuracy, thermal camera geometric calibration accuracy, thermal camera and motion tracking accuracy). After taking a close look at the individual accuracies, a global measure for the 3D thermal mapping accuracy needs to be found and evaluated. Second, in a mapping, new IR or US data is simply added by creating a new 3D point with assigned data. In order to better evaluate the 3D map it would be necessary to compact the data and merge points from different observations that are close to each other. Multiple (temperature) values could be replaced by the statistics (mean value and variance) estimated from the multiple measurements. Finally, a tracking system more portable than the Vicon could be used to favor the transition to a system that can be used outside the lab with minimum setup time. The tracking system should further allow the user to move the system (with overlap) to inspect a scene with a volume that is larger than the tracking volume. Experiments exploiting the (portable) tracking system used for estimating the pose of a 3D handheld scanner (Creaform's CTrack and Metrascan) are currently being conducted and show promising results for indoor experiments.

\section{Acknowledgements}

The research presented in this paper was supported by the NSERC-Creaform Industrial Research Chair on 3D scanning grant. The authors thank M. Denis Ouellet for his engineering support during the data collection experiments and system deployment.

\section{REFERENCES}

[1] Zhang, Z., "A Flexible New Technique for Camera Calibration," IEEE Transactions on Pattern Analysis and Machine Intelligence, vol. 22, no. 11, pp. 1330-1334, 2000.

[2] http://www.creaform3d.com/en/metrology-solutions/handheld-portable-3d-scanner-goscan-3d

[3] Soldan, S., "Towards 3D thermal imaging: Geometric calibration of thermal imaging cameras and registration with 3D surface models using optical motion tracking for camera and object position," Technical Report, University of Kassel, Germany and Laval University, Quebec, April 2013, 22 p.

[4] OpenCV, "Open Source Computer Vision Library," 2013, accessed 17. April 2013. [Online]. Available: http://opencv.org/

[5] J.-Y. Bouguet, "Camera Calibration Toolbox for Matlab," 2013, accessed 17. April 2013. [Online]. Available: http://www.vision.caltech.edu/bouguetj/calib_doc/

[6] Kitware Inc, "File Formats for VTK Version 4.2," 2013, accessed 17. April 2013. [Online]. Available: http://www.vtk.org/VTK/img/file-formats.pdf

[7] [6] Kitware Inc, "ParaView - Open Source Scientific Visualization," 2013, accessed 17. April 2013. [Online]. Available: http://www.paraview.org/

[8] [7] Kitware Inc, "KiwiViewer," 2013, accessed 17. April 2013. [Online]. Available: http://www.kiwiviewer.org/ 